\title{
Correlated Imaging of Topology and Composition Within Phase-separated Supported Lipid Membranes
}

Mary Kraft ${ }^{1}$, Christopher Anderton ${ }^{2}$ and Peter Weber ${ }^{3}$

${ }^{1}$ University of Illinois at Urbana-Champaign, Urbana-Champaign, Illinois, United States, ${ }^{2}$ Pacific Northwest National Lab, Richland, Washington, United States, ${ }^{3}$ Lawrence Livermore National Laboratory, Livermore, California, United States

Compartmentalization of the plasma membrane into domains of differing composition is required for proper cell function. Phase-separated supported lipid bilayers are often used as simplified models for probing the physical interactions that give rise to lipid distribution within cellular membranes. Though these model systems enable precise control of the lipid species within the membrane, imaging lateral variations in phase behavior and composition within a model membrane is challenging because such information cannot be acquired with a single technique.

In this study, we use correlated imaging with complementary techniques to characterize phase-separated supported lipid membranes. Specifically, atomic force microscopy (AFM) was used to acquire information about the phase behavior at specific locations within the membrane, and the same location in the membrane was imaged with secondary ion mass spectrometry performed with a Cameca NanoSIMS 50 to determine the lipid composition in each phase. The NanoSIMS 50 images the elemental and isotopic composition in a sample with a lateral resolution as high as $50 \mathrm{~nm}$ [1]. To enable identifying each lipid species according to the monoatomic and diatomic secondary ions it produces using a NanoSIMS instrument, each lipid species in the phase-separated lipid membrane was labeled with a different rare stable isotope. A small fraction of the lipids in the membrane was also labeled with fluorophores so that fluorescence microscopy could be used to rapidly assess phase-separation and sample quality. To facilitate imaging the same region of the membrane with multiple complementary techniques, the supported lipid membranes were prepared on substrates patterned with a chrome grid that served as fiducial markers. Because NanoSIMS analysis is performed under ultra-high vacuum, the membranes were dehydrated without perturbing the lipid distribution within them by flash-freezing and sublimation of the resulting ice at low temperature and pressure. After mapping the fluorescent lipid distribution in the supported membrane sample, AFM imaging was performed on the well-preserved regions of the membrane. Finally, the NanoSIMS 50 was used to image the secondary ion signals characteristic of each labeled species at the same region on the supported lipid membrane.

We used this correlative fluorescence microscopy, AFM and SIMS imaging of lipid phase-separation, packing, and composition to study the distribution of cholesterol within supported lipid membranes. The lipid membrane was comprised of a saturated ${ }^{13} \mathrm{C}$-labeled lipid $\left({ }^{13} \mathrm{C}\right.$-DSPC), an unsaturated ${ }^{15} \mathrm{~N}$-labeled lipid $\left({ }^{15} \mathrm{~N}\right.$-DOPC), and cholesterol. At room temperature, phase separation between the ${ }^{13} \mathrm{C}-\mathrm{DSPC}$ and ${ }^{15} \mathrm{~N}$-DOPC occurs, but the distribution of cholesterol between the phase-separated lipids had not been determined. Correlative imaging with AFM and SIMS revealed the cholesterol was nearly equally distributed between the membrane regions enriched with ${ }^{13} \mathrm{C}$-DSPC or ${ }^{15} \mathrm{~N}$-DOPC. This demonstrates that additional information can be acquired by correlative imaging with complementary techniques.

\section{References}


1. L. Gorman and M. K. Kraft, "High-Resolution Secondary Ion Mass Spectrometry Analysis of Cell Membranes," Analytical Chemistry, 2020, 92 (2), 1645-1652.

2. C. R. Anderton, P. K. Weber, K. Lou, I. D. Hutcheon, and M. L. Kraft, "Correlated AFM and NanoSIMS imaging to probe cholesterol-induced changes in phase behavior and non-ideal mixing in ternary lipid membranes," Biochimica et Biophysica Acta, Biomembranes, 2011, 1808 (1), 307-315. 